\title{
Utilisation de bactériocines pour la production d'aliments plus sûrs : mythe ou réalité ?
}

\author{
J Richard \\ Unité de recherches laitières, Inra, 78352 Jouy-en-Josas, France
}

\begin{abstract}
Résumé - Le présent article est une synthèse de la communication présentée lors du colloque du Club des bactéries lactiques tenu à Paris en septembre. II débute par une analyse critique des résultats de détermination des $\mathrm{CMI}$ (concentrations minimales inhibitrices) effectuées dans différents laboratoires, ainsi que des résultats d'expériences démontrant l'activité de deux bactériocines de bactéries lactiques, la nisine et une pédiocine, dans deux types d'aliments. Puis on montre, dans le cas d'un processus faisant intervenir une fermentation (en l'occurence, la fabrication de fromages à pâte molle et croûte moisie) comment on peut améliorer l'activité d'une bactériocine (la nisine) jusqu'à obtenir la quasiéradication des bactéries indésirables (les Listeria) grâce à une utilisation raisonnée d'un système inhibiteur impliquant une bactériocine. Deux conclusions principales sont tirées de cette synthèse : 1)l'utilité des bactériocines pour la maîtrise des flores indésirables dans les aliments et 2) la nécessité de poursuivre des recherches sur les conditions de leur activité in situ, en complément de celles beaucoup plus avancées concernant leur mode d'action, la relation structure-fonction et l'organisation des gènes codant pour leur production.
\end{abstract}

bactériocine / nisine / pédiocine / Listeria / inhibition / fromage

Summary - Use of bacteriocins for a surer production of food products: myth or reality? The present paper is a shortened version of a communication at the Club des Bactéries Lactiques held in Paris last September. Firstly, a critical analysis of the determination of MICs (minimum inhibitory concentrations) is presented along with results demonstrating the efficacy of two bacteriocins (nisin and a pediocin) in two kinds of foods, dairy and meat products. Then, we describe the results of experiments conducted in our laboratory on inhibition of Listeria monocytogenes in Camembert cheese made using a nisin-positive starter in comparison with a control made with a nisin-negative starter. We show that inhibition by nisin can be considerably improved by both adding small amounts of this compound to cheese milk when $\mathrm{pH}$ is most appropriate to nisin activity, and heating milk at a sublethal temperature to Listeria, which is thought not to affect the technological properties of cheese milk. It results that complete eradication of Listeria in soft surface-ripened cheeses could be achieved if raw milk is produced under good hygienic conditions. Two main conclusions are drawn from this presentation: i) the bacteriocins are very useful to control undesirable bacteria in foods; and ii) research on application must be pursued in complement to that on genetics and mode of action of bacteriocins.

bacteriocin / nisin / pediocin / Listeria / inhibition / cheese 


\section{INTRODUCTION : NÉCESSITÉ D'UNE AMÉLIORATION \\ DE LA QUALITÉ SANITAIRE ET DE LA DURÉE DE CONSERVATION DE CERTAINS ALIMENTS}

On assiste, depuis quelques années, à un développement extraordinaire des connaissances de base sur les bactériocines de bactéries lactiques, que ce soit sur le plan de leur mode d'action et depuis peu, de la relation entre leur structure et leur fonction, ou sur le plan de l'organisation des gènes impliqués dans leur production. En regard de ces travaux d'un haut niveau scientifique, l'approche plutôt rudimentaire des travaux consacrés à l'activité de certaines bactériocines dans divers aliments peut surprendre. On se contente généralement de montrer que l'addition de quantités variables de ces substances entraîne la perte de viabilité d'une partie de la population bactérienne cible, en général des listeria. Bien que les facteurs qui affectent l'activité d'une bactériocine soient connus de façon générale, un utilisateur éventuel ne peut déduire des expériences décrites, si la bactériocine utilisée l'est dans des conditions satisfaisantes, ou si son efficacité peut être grandement améliorée. II n'est pas indiqué non plus en quoi la nouvelle bactériocine présente un avantage sur les substances de même nature décrites auparavant, la nisine en particulier.

Malgré la découverte d'un certain nombre de bactériocines actives vis-à-vis de bactéries indésirables dans les aliments, en particulier vis-à-vis de Listeria monocytogenes, la nisine reste la seule substance antibactérienne utilisée industriellement, et encore, son utilisation semble limitée à quelques aliments, comme les fromages fondus dans lesquels elle empèche le gonflement dû au développement de Clostridia (Delves-Broughton, 1990).
Les problèmes soulevés par la présence éventuelle des Listeria dans les aliments d'origine animale expliquent sans doute l'importance des travaux réalisés aux ÉtatsUnis sur l'activité des bactériocines les plus intéressantes dans divers aliments, en particulier dans la viande et dans des produits carnés élaborés. S'ajoute à cela la tendance à augmenter la durée de commercialisation de certains d'entre eux tout en réduisant l'usage d'agents antibactériens chimiques (nitrites, par exemple). En France, cette nécessité d'utiliser des agents antibactériens naturels ne semble pas avoir été perçue avec autant d'acuité, malgré les problèmes sanitaires posés par les fromages au lait cru et des épisodes de listériose provoquée par divers aliments. Est-ce dû au fait que les résultats des études sur l'efficacité des bactériocines ne sont pas toujours très convaincants (pour ne pas dire source de perplexité de la part d'un lecteur peu averti, mais attentif), ou faciles à appliquer ? Ou bien, les industriels de l'agro-alimentaire français sont-ils réticents à changer leurs habitudes, ou craignent-ils que l'utilisation de ces agents antibactériens ne soit finalement pas autorisée?

Dans cet article, nous commencerons par un commentaire des résultats de la détermination de la concentration minimale inhibitrice ( $\mathrm{CMI}$ ) qui peuvent être difficiles à interpréter pour un technologue. Puis après avoir donné quelques exemples de l'activité dans deux types d'aliments des deux bactériocines les plus étudiées à ce jour, la nisine produite par certaines souches de l'espèce Lactococcus lactis subsp lactis, et la pédiocine produite par une souche de l'espèce Pediococcus acidilactici, nous montrerons comment on peut améliorer l'efficacité d'une bactériocine (en la circonstance, la nisine) par une prise en compte des facteurs qui affectent son activité au cours d'un processus technologique complexe, puisqu'il met en œuvre une fermentation. 


\section{ÉVIDENCE DE L'EFFICACITÉ DE QUELQUES BACTÉRIOCINES DE BACTÉRIES LACTIQUES DANS DIVERS ALIMENTS}

Une des étapes-clés dans la caractérisation d'une bactériocine consiste à établir son spectre d'activité en utilisant diverses techniques, et une collection de souches cibles appartenant à divers genres ou espèces. Le plus souvent, pour des raisons de simplicité, on se contente de quelques souches par espèce et l'expérience est conduite avec une concentration unique et élevée de bactériocine, de sorte que les résultats sont de nature «tout ou rien». La détermination de la concentration minimale inhibitrice (CMI) permet de quantifier cet effet, et de mettre en évidence des groupes de bactéries plus sensibles les uns que les autres, ce qui est de première importance au plan de l'application.

En fait, on note, d'un auteur à l'autre, de grandes divergences quant aux milieux de culture utilisés, la taille de la population bactérienne et son état physiologique (bactéries en phase exponentielle ou en phase stationnaire), ainsi que la température à laquelle le test est conduit. II est dommage en outre, que dans ces déterminations on n'utilise pratiquement jamais une bactériocine connue (par exemple, la nisine A, disponible dans le commerce) et la même souche cible comme références.

Le tableau I présente comme exemple une des premières publications sur l'activité de la nisine envers Listeria monocytogenes. On constate une différence considérable dans les estimations de la CMI selon la souche et surtout, le milieu de culture utilisé pour déterminer les CMI. Si l'on prend en compte une production maximale de nisine de $2000 \mathrm{UI} / \mathrm{ml}$ dans un milieu approprié et des conditions optimales de culture d'une souche de lactocoque nisinepositive, force nous est de conclure des résultats précédents que la CMI pour la souche de listeria la plus résistante ne peut être atteinte dans un aliment que par addition de la substance purifiée ou d'une culture très concentrée. Une publication plus récente (Hugenholtz et de Veer, 1991) fait état de CMI de nisine vis-à-vis de $L$ monocytogenes considérablement plus faibles que dans le tableau précédent, soit de 8 à $80 \mathrm{UI} / \mathrm{ml}$. Nos propres estimations donnent

Tableau I. Variation de la sensibilité à la nisine de quelques souches de Listeria (adapté de Benkerroum et Sandine, 1988).

Variation in susceptibility to nisin within strains of Listeria (adapted from Benkerroum and Sandine, 1988).

Espèces et souches

Concentration minimale inhibitrice (Ul/ml)

Sur milieu TSA

Sur milieu MRS

Listeria monocytogenes

Scott A

V7

15313

35152

$7644 \mathrm{~K}$

Listeria ivanovii C194

Listeria saligeri LA15
$1,2 \times 10^{4}$
$1,2 \times 10^{5}$
$1,5 \times 10^{4}$
$1,5 \times 10^{4}$
$1,5 \times 10^{4}$
$7,4 \times 10^{4}$
$1,5 \times 10^{4}$

$1,7 \times 10^{3}$

$3,4 \times 10^{3}$

$1,7 \times 10^{3}$

3,7

37

37

37 
des valeurs plus élevées, voisines de $500 \mathrm{UI} / \mathrm{ml}$ pour un ensemble de souches de listeria (Maisnier-Patin, 1994). Ces divergences peuvent être attribuées aux conditions de détermination de la CMI, en particulier, au milieu de culture utilisé.

Quoi qu'il en soit, d'un point de vue pratique, il est intéressant de savoir si la CMI doit être atteinte dans un aliment particulier pour assurer un résultat satisfaisant, et dans ce cas, si cette concentration peut être produite in situ par une souche productrice de bactériocine, ou si cette substance doit impérativement être ajoutée. Dans ce cas, en dehors d'une question réglementaire se posera une question de coût.

La plupart des publications montrant l'activité d'une bactériocine dans un produit alimentaire s'appuient sur des expériences dans lesquelles on ajoute un filtrat de culture ou la bactériocine plus ou moins purifiée, généralement à des concentrations bien inférieures à la CMI de la souche test, et on observe la perte de viabilité d'une bactériecible ou plus rarement d'un cocktail de souches de même espèce. Pour faciliter les examens bactériologiques, l'aliment est généralement inoculé à haut niveau. De cette façon on évite de devoir faire la détection des bactéries survivantes par la technique lente, laborieuse et de sensibilité réduite qui consiste à faire croître ces bactéries dans un milieu d'enrichissement approprié avant de les mettre en évidence sur un milieu sélectif. Souvent, on est également obligé d'identifier les bactéries qui apparaissent pour éviter de les confondre avec d'autres qui se seraient également développées dans le milieu d'enrichissement.

La figure 1 montre, comme exemple de cette démarche, l'activité de la nisine vis-àvis d'une souche de listeria ajoutée à de la viande fraîche hachée et conservée à basse temperature ( $M$ Murray, communication personnelle). Les concentrations

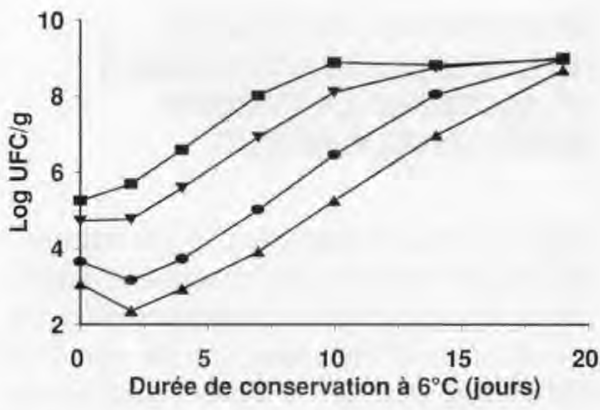

Fig 1. Inhibition de Listeria innocua (souche Lin11) par la nisine dans de la viande de porc hachée conservée à $6^{\circ} \mathrm{C}$ (Numération des listeria sur milieu Palcam). : témoin sans nisine; essai avec $1000(\mathbf{\nabla}), 5000(\bullet)$ et $10000(\mathbf{\Delta})$ $\mathrm{UI} / \mathrm{ml}$ de nisine dans le liquide intersticiel.

Inhibition of Listeria innocua (strain Lin11) by nisin in ground pork meat held at $6^{\circ} \mathrm{C}$ (Listeria enumeration on Palcam). control without nisin; experiment with $1000(\boldsymbol{\nabla}) ; 5000(\bullet) ; 10000$ (A) IU of nisin per $\mathrm{ml}$ of liquid phase.

indiquées sont celles de la phase intersticielle, c'est-à-dire de la solution de bactériocine en contact avec la surface de la viande avant hachage. On observe, par rapport au témoin sans nisine, une perte de viabilité des bactéries cibles qui se poursuit pendant 2 jours, suivie d'une croissance des celluiles survivantes à la même vitesse que les bactéries dans la viande témoin. On notera que $1000 \mathrm{UI} / \mathrm{ml}$ de nisine, soit deux fois la CMI de cette souche déterminée au laboratoire, n'entraîne qu'une diminution modeste de la souche cible. II faudrait plus de $5000 \mathrm{UI} / \mathrm{ml}$ pour obtenir 3 unités de log de réduction de cette bactérie, soit une destruction de $99,9 \%$ de la population initiale.

La figure 2 illustre le comportement de la même souche de Listeria en présence de nisine dans du lait écrémé et dans un milieu de laboratoire, les deux milieux étant à $30^{\circ} \mathrm{C}$. II est remarquable qu'il faut beaucoup moins de nisine dans le lait que dans la viande pour obtenir 2 unités de log de 


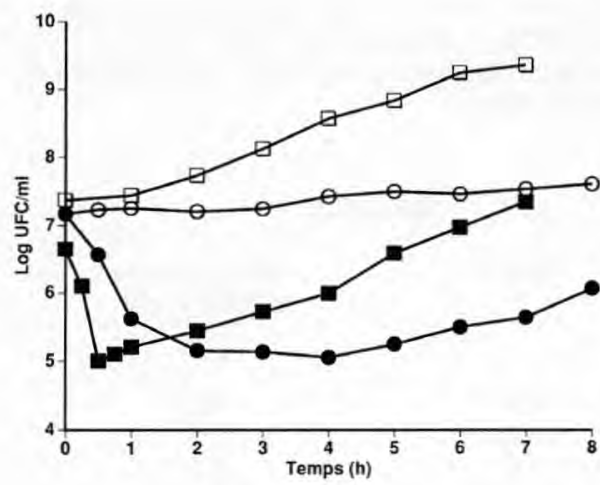

Fig 2. Comportement de Listeria innocua (souche Lin11) à $30^{\circ} \mathrm{C}$ dans le milieu TSBYE (Tryptic Soy Broth+Yeast Extract) sans nisine ( $\square$ ) ou

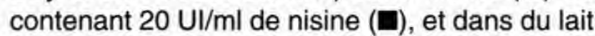
écrémé sans nisine $(O)$ ou contenant $50 \mathrm{UI} / \mathrm{ml}$ de nisine (•).

Fate of Listeria innocua (strain Lin11) at $30^{\circ} \mathrm{C}$ in tryptic soy broth plus yeast extract (TSBYE) without nisin (D) or in presence of $20 \mathrm{IU} / \mathrm{ml}$ of nisin $(\boldsymbol{\square})$ and in skim milk without nisin $(O)$ or containing $50 \mathrm{IU} / \mathrm{ml}$ of nisin (๑). réduction de la population initiale (soit $99 \%$ de destruction de cette population), et que pour atteindre ce taux de réduction il faut deux fois et demi plus de nisine dans le lait que dans le milieu de laboratoire TSBYE. On notera aussi que la perte de viabilité des Listeria est nettement plus rapide dans le TSBYE que dans le lait. Cela est dû au fait que le TSBYE est plus favorable à la croissance des Listeria que le lait, ce qui entraîne une plus grande sensibilité de ces bactéries à la nisine.

Les tableaux II et III donnent l'exemple de résultats obtenus aux États-Unis à 5 ans de distance, montrant l'efficacité dans deux types de produits alimentaires d'une pédiocine, la bactériocine produite par deux souches différentes de l'espèce Pediococcus acidilactici. La pédiocine produite par les deux souches porte des noms différents, mais il s'agit exactement de la même molécule, un peptide de 44 acides aminés présentant la même séquence (Jack et al, 1995). On constate dans les deux cas, une diminution de la teneur des produits en Lis-

Tableau II. Effet de 100 unités arbitraires (UA) de pédiocine PA.1 par gramme sur Listeria monocytogenes $\left(\mathrm{CMl}: 55 \mathrm{UA} / \mathrm{ml}\right.$ ) ajoutés à différents produits laitiers conservés à $4{ }^{\circ} \mathrm{C}$ (d'après Pucci et al, 1988).

Effect of 100 arbitrary units (AU) per gram of pediocin PA.1 on Listeria monocytogenes (MIC: 55 $A U / \mathrm{ml}$ ) in different dairy products stored at $4^{\circ} \mathrm{C}$ (adapted from Pucci et al, 1988).

Purée d'incubation
(jours)

Crême half-and-half (18\% de $M G$, $\mathrm{pH} 6,6)$

Soupe au Cheddar

( $1 \%$ de sel, $\mathrm{pH} 6,0)$
Nombre de listeria $\left(\log _{10}\right.$ ufc/g)

Témoin avec $100 \mathrm{UAVg}$ de pédiocine


Tableau III. Effet combiné de la pédiocine $\mathrm{AcH}(5000 \mathrm{UA} / \mathrm{ml})$ et du diacétate de sodium sur Listeria monocytogenes dans du jus de filet de dinde conservé à $4{ }^{\circ} \mathrm{C}$ (d'après Schlyter et al, 1993).

Combined effect of pediocin $\mathrm{AcH}(5,000 \mathrm{AU} / \mathrm{ml})$ and sodium diacetate (*) on Listeria monocytogenes in turkey slurries held at $4^{\circ} \mathrm{C}$ (adapted from Schlyter et al, 1993).

\begin{tabular}{|c|c|c|c|}
\hline \multirow[t]{2}{*}{ Ajout } & \multirow{2}{*}{$\begin{array}{c}\text { Durée } \\
\text { d'incubation } \\
\text { (jours) }\end{array}$} & \multicolumn{2}{|c|}{$\log _{10}$ ufc/g } \\
\hline & & Témoin & $\begin{array}{l}\text { Avec pédiocine } \\
\text { (5 000 UA/ml) }\end{array}$ \\
\hline aucun & 0 & 4,5 & 3,6 \\
\hline \multirow[t]{3}{*}{$(\mathrm{pH} 6,2)$} & 7 & 6,2 & 3,3 \\
\hline & 14 & 7,8 & 4,2 \\
\hline & 28 & 8,2 & 8,0 \\
\hline & 0 & 4,5 & 2,8 \\
\hline \multirow{3}{*}{$(\mathrm{pH} 5,2)$} & 7 & 4,0 & 2,1 \\
\hline & 14 & 3,8 & 1,4 \\
\hline & 28 & 3,8 & 1,1 \\
\hline
\end{tabular}

teria suivie, après une semaine ou deux, par une croissance de cette flore, sauf dans le cas du produit additionné de "diacétate" (un composé solide générant de l'acide acétique par dilution dans l'eau) : ce composé à lui-seul entraîne une perte de viabilité des listeria qui s'accentue en présence de pédiocine.

Ces quelques exemples montrent l'efficacité dans des aliments différents et des conditions diverses de conservation des deux bactériocines de bactéries lactiques les plus étudiées pour l'instant. Toutefois, le taux de destruction dépend non seulement de la concentration du produit en bactériocines, mais du produit lui-même, de la nature d'additifs éventuels, et de la température à laquelle il est conservé. Dans tous les cas, on observe une reprise de croissance des bactéries survivantes. Ce dernier point, d'une extrême importance pratique et théorique, mériterait un long développement. Nous nous bornerons à un commentaire particulier.

Pour des raisons pratiques évoquées plus haut, les études ont été réalisées sur des aliments artificiellement contaminés et cela à des niveaux très exagérés par rapport à la réalité. Si les bactéries indésirables dans les produits alimentaires sont naturellement dans le même état de sensibilité visà-vis de ces bactériocines que les souches utilisées dans ces études, on peut raisonnablement s'attendre à ce que le taux de destruction observé avec des niveaux élevés soit atteint avec des niveaux beaucoup plus faibles. On peut s'attendre même à ce qu'il soit dépassé. Les expériences réalisées en vraie grandeur sur des aliments permettent donc de déterminer la concentration de bactériocine, et les conditions de son utilisation, assurant un taux de destruction suffisant pour entraîner la disparition de la flore indésirable. Cette concentration 
peut être bien inférieure à la CMI déterminée dans un milieu de culture de laboratoire. Nous présenterons plus loin un exemple concret de cette approche. Pour l'instant donnons en exemple un aliment contenant moins de une bactérie indésirable par gramme. Si on a trouvé expérimentalement (avec une contamination artificielle à haut niveau) une concentration de bactériocine entraînant une chute de population de 3 unités de log, alors nous pouvons pénser que dans les conditions normales de contamination de cet aliment, nous retrouverons moins d'une bactérie indésirable par $\mathrm{kg}$ de ce produit. La vérification expérimentale de cette assertion est évidemment difficile, puisque le niveau des bactéries survivantes est largement en dessous de la limite habituelle de détection d'un contaminant (quelques cellules dans $25 \mathrm{~g}$ ).

\section{PROBLÈMES POSÉS PAR}

\section{L'UTILISATION D'UNE BACTÉRIOCINE, OU DE LA SOUCHE LA PRODUISANT, AU COURS D'UN PROCESSUS TECHNOLOGIQUE REPOSANT SUR UNE FERMENTATION}

Le cas des fromages à pâte molle et croûte moisie (type Camembert) fabriqués avec du lait cru fournit un modèle intéressant (et d'actualité) d'une démarche raisonnée dans l'utilisation de bactériocines en vue de détruire les listeria que ce lait pourrait contenir.

Dans un premier temps, nous avons fabriqué des fromages dans notre unité pilote, à partir de lait pasteurisé artificiellement contaminé en listeria (Maisnier-Patin et al, 1992). Nous avons choisi la souche V7 de $L$ monocytogenes en raison de sa bonne tenue au cours de la fabrication de fromages de ce type (Ryser et Marth, 1987). Trois niveaux de contamination du lait ont été expérimentés : $10,10^{3}$ et $10^{5}$ cellules par $\mathrm{ml}$ de lait. Ces bactéries étaient en phase stationnaire (après $18-24 \mathrm{~h}$ de culture à $30^{\circ} \mathrm{C}$

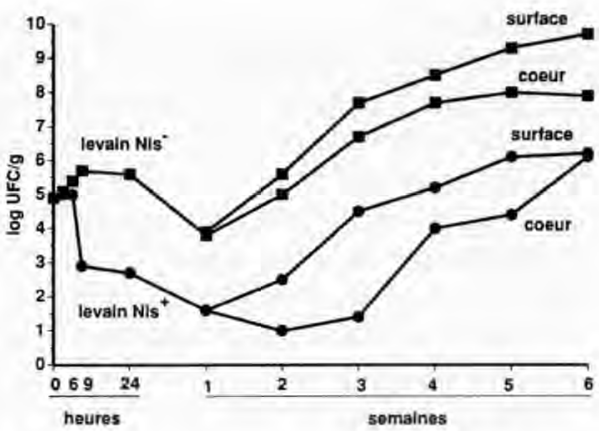

Fig 3. Comportement de Listeria monocytogenes (souche V7) au cours de la fabrication de fromages à pâte molle et croûte fleurie à l'aide d'un levain non producteur de nisine (Nis') et d'un levain producteur de nisine $\left(\mathrm{Nis}^{+}\right)$.

Fate of Listeria monocytogenes (strain V7) during making Camembert cheese with a nisin-producing starter (Nis ${ }^{+}$) and a Nis- starter as control.

sur lait), donc dans un état physiologique assurant une moins grande sensibilité à la nisine que si elles étaient en phase exponentielle de croissance, Les fromages expérimentaux étaient fabriqués avec une souche de lactocoque productrice de nisine, et les fromages témoins avec une souche de même espèce, mais nisine-négative. L'évolution du nombre de Listeria dans le lait et les fromages était suivie par dénombrement sur un milieu sélectif dont on a vérifié l'innocuité vis-à-vis des Listeria présentes dans ces fromages.

La figure 3 illustre le comportement de $L$ monocytogenes dans les deux types de fromages au cours de leur fabrication et de leur affinage. L'évolution de la souche cible dans les fromages témoins fabriqués avec la souche de lactocoque nisine-négative était tout à fait similaire à celle décrite par Ryser et Marth. Dans les fabrications avec la souche productrice de nisine, cette substance était produite entre la $6^{\mathrm{e}}$ et la $9^{\mathrm{e}}$ heure, ce qui correspondait à la deuxième moitié de la phase de croissance des lactocoques, et atteignait 700 à $800 \mathrm{UI} / \mathrm{g}$ dans 
les fromages au démoulage. Ensuite, sa concentration s'abaissait rapidement à la surface des fromages, du fait probablement de la dégradation de la bactériocine par les protéases produites par le pénicillium, et plus lentement dans la masse. Malgré la production de nisine une fois et demie supérieure à la $\mathrm{CMI}$ de la souche de Listeria utilisée (environ $500 \mathrm{UI} / \mathrm{ml}$ ), on n'observait que 2 unités de log de réduction de cette bactérie, soit l'équivalent de $50 \mathrm{Ul} / \mathrm{ml}$ de nisine ajoutée à du lait maintenu à $30^{\circ} \mathrm{C}$ (fig 2). Malgré cette faible activité de la nisine produite au cours de la fabrication des fromages, une différence de 2,4 unités de log (moyenne de trois expériences différentes) était maintenue pendant toute la durée d'affinage entre les fromages fabriqués avec la souche $\mathrm{Nis}^{+}$et les témoins, soit une réduction à $1 / 200$ du niveau des listeria dans les fromages. Avec un niveau de contamination du lait en Listeria 100 fois plus faible (soit $10^{3}$ cellules par $\mathrm{ml}$ ), on obtenait des résultats encore meilleurs, puisqu'on ne retrouvait pas de Listeria dans les fromages affinés, alors que par extrapolation des résultats avec le niveau de contamination le plus élevé, on aurait dû en avoir au moins $10^{3}$ par gramme en fin d'affinage. Avec le niveau de contamination le plus faible (10 cellules de listeria par $\mathrm{ml}$ ), se posait un problème de détection dủ au très faible niveau des bactéries survivantes (absence probable dans $100 \mathrm{~g}$ de fromage affiné).

Nous avons montré par ailleurs que la cause principale de la faible activité de la nisine produite au cours de la fabrication de ce type de fromage résultait d'une perte d'efficacité de cette bactériocine dans le lait à $\mathrm{pH}$ acide. Ce phénomène non encore expliqué n'est cependant pas commun à toute bactériocine. En effet, on a observé dans notre laboratoire que la pédiocine $\mathrm{AcH}$ produite par Pediococcus acidilactici F (Bhunia et al, 1988) gardait et même renforçait son activité dans le lait acide. À l'inverse de la nisine, la mésentéricine $Y 105$ produite par une souche de Leuconostoc mesenteroides (Héchard et al, 1992) n'était active que dans le lait acide. Le fait que la nisine résiduelle dans le cceur des fromages (environ 200 UI/g à la $6^{e}$ semaine) n'entraîne pas de chute de population lorsque le $\mathrm{pH}$ remonte peut être expliqué d'une part par une adsorption irréversible de la bactériocine sur les composants du fromages et d'autre part, par une résistance accrue à la nisine des cellules survivantes.

Puisque la nisine est produite tardivement dans le lait et que son efficacité diminue quand celui-ci s'acidifie, une façon toute simple d'améliorer les performances de cette substance paraissait être de l'ajouter dès le début de la fabrication des fromages. Nous avons vérifié que dans les conditions de son utilisation comme levain, notre souche $\mathrm{Nis}^{+}$était capable de résister jusqu'à $500 \mathrm{UI} / \mathrm{ml}$ de cette bactériocine. L'addition de seulement $50 \mathrm{UI} / \mathrm{ml}$ de lait (soit environ $1 \mathrm{mg}$ de substance pure par litre), permettait une destruction supplémentaire de 1 unité de log des listeria dans les fromages fabriqués avec la souche nisine-positive. Nous avons montré par ailleurs (Maisnier-Patin et al, 1995) qu'un chauffage du lait à une température sublétale pour les Listeria (par exemple quelques minutes à $54^{\circ} \mathrm{C}$ ) entraînait une plus grande sensibilité de ces bactéries vis-à-vis de la nisine.

D'après divers auteurs (Beckers et al 1987 ; Lovett et al, 1987 ; Fenlon et Wilson, 1989 ; Rohrbach et al, 1992 ; Peeler et Bunning, 1994), moins de $10 \%$ des échantillons de lait cru prélevé à la ferme contiendraient $L$ monocytogenes, et le niveau de contamination du lait des échantillons positifs serait généralement inférieur à 1 listeria par $\mathrm{ml}$. On peut déduire de ces observations un niveau de contamination d'un lait de mélange inférieur à 100 listeria par litre. Nous avons montré par ailleurs (Siswanto et Richard, 1992) que les listeria ne se développent pas dans le lait conservé moins de 2 jours à basse température, ni au cours de 
Tableau IV. Probabilité de trouver une listeria dans $100 \mathrm{~g}$ de fromage à pâte molle âgé d'une semaine, en fonction du niveau de contamination du lait et de la technologie fromagère mise en œuvre.

Probability to find one Listeria in $100 \mathrm{~g}$ of soft cheese aged 1 week as a function of milk contamination and cheese technology.

\begin{tabular}{|c|c|c|c|c|c|}
\hline \multirow[t]{2}{*}{ Levain utilisé } & \multirow{2}{*}{$\begin{array}{l}\text { Nisine ajoutée } \\
(\mathrm{mg} / \mathrm{l})\end{array}$} & \multirow{2}{*}{$\begin{array}{l}\text { Perte de viabilité } \\
\quad\left(\log _{10} \text { ufc/g) }\right.\end{array}$} & \multicolumn{3}{|c|}{ Nombre de listeria par litre de lait } \\
\hline & & & 1 & 10 & 100 \\
\hline Ordinaire & 0 & 1,7 & $2.10^{-2}$ & $2.10^{-1}$ & 1 \\
\hline $\begin{array}{l}\text { Producteur de nisine } \\
\text { Sans chauffage } \\
\text { Chauffage modéré }\end{array}$ & $\begin{array}{l}0 \\
1 \\
1\end{array}$ & $\begin{array}{l}4,1 \\
5,1 \\
8,1\end{array}$ & $\begin{array}{l}10^{-4} \\
10^{-5} \\
10^{-8}\end{array}$ & $\begin{array}{l}10^{-3} \\
10^{-4} \\
10^{-7}\end{array}$ & $\begin{array}{l}10^{-2} \\
10^{-3} \\
10^{-6}\end{array}$ \\
\hline
\end{tabular}

la fermentation lactique, du fait d'abord d'une compétition avec les lactocoques pour des nutriments puis de l'abaissement du $\mathrm{pH}$ du lait (Siswanto, 1993). Admettons, pour simplifier, que le rendement en fromage frais soit de $10 \%$ (100 g par litre) et que toutes les listeria présentes dans le lait soient retenues dans le caillé. Avec du lait contenant 100 Listeria par litre, on aurait alors une listeria par gramme de fromage frais. Les résultats de nos expériences avec le levain nisine-négatif font apparaître, au cours de la première semaine, une perte moyenne de viabilité des Listeria (due à un séjour prolongé en présence d'acide lactique) de 1,7 unité de log, soit une division par 50 du niveau de cette population. En mettant en $œ u v r e$ du lait contenant 100 Listeria par litre, on devrait donc trouver deux Listeria dans $100 \mathrm{~g}$ de fromage fabriqué avec un levain non producteur de nisine, soit la certitude (probabilité égale à 1) d'en trouver

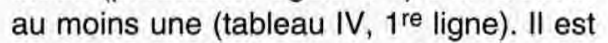
évident que si le lait est produit avec une hygiène renforcée, on peut s'attendre à un niveau de contamination en listeria 10 à 100 fois plus faible, ce qui est très appréciable, mais peut-être difficile à obtenir. D'après nos expériences, l'utilisation d'un levain producteur de nisine devrait diviser ces probabilités au moins par 200 (écart moyen de
2,4 unités de log au démoulage entre fromages témoins et fromages expérimentaux). L'addition de $50 \mathrm{UI} / \mathrm{ml}$ de nisine au lait de fabrication entraînerait encore une diminution du risque d'un facteur 10 (tableau IV, $3^{e}$ ligne). En combinant, a) l'addition d'une petite quantité de nisine au lait, b) le chauffage du lait à une température modérée respectant au mieux ses propriétés technologiques etc. l'utilisation d'un levain producteur de nisine, on devrait pouvoir réduire par un facteur au moins égal à 1 million, le risque de trouver une listeria par $100 \mathrm{~g}$ de fromage fabriqué avec du lait contenant 100 listeria par litre (tableau IV, 4 e ligne). Cette démarche associée à un renforcement de l'hygiène à la production permettrait alors la quasi-éradication des Listeria présentes dans le lait cru. Son inconvénient majeur réside dans le fait que les bactéries lactiques autres que les lactocoques éventuellement présentes dans le levain seraient également inhibées. En outre, les fromages ne pourraient plus bénéficier de l'appellation "au lait cru" porteuse d'une promesse de qualité gustative particulière.

Une alternative consisterait à utiliser une bactériocine n'ayant pas d'activité sur la flore lactique. Nous disposons déjà de souches de bactéries lactiques dont la bactériocine satisfait cette exigence. Certaines 
ne sont pas capables de pousser dans le lait en présence des lactocoques. II faudrait donc les faire pousser à part, en tant que levain séparé, et en introduire dans le lait une quantité suffisante pour que la bactériocine y soit apportèe à une concentration satisfaisante. II est évident qu'il serait plus simple d'utiliser la bactériocine purifiée selon un procédé industriel adéquat, comme cela se pratique avec la nisine.

Une autre possibilité est de transférer, dans les bactéries utiles autres que les lactocoques producteurs de nisine, les gènes codant pour la résistance des souches productrices de nisine (gènes d' "immunité") ou de souches naturellement résistantes à la nisine. C'est une voie longue et si elle réussit, posera le problème de l'autorisation d'utiliser des bactéries génétiquement modifiées contenant de l'ADN hétérologue.

\section{CONCLUSIONS ET PERSPECTIVES}

On dispose actuellement d'un ensemble de souches de bactéries lactiques productrices de bactériocines ayant des propriétés différentes. En supposant levées toutes les limitations réglementaires relatives à leur emploi en tant qu'additif, le problème serait de choisir la bactériocine la plus appropriée à une utilisation particulière, c'est-à-dire celle dont l'efficacité vis-à-vis des bactéries cibles est la plus grande, sans que la flore utile dans certains produits soit affectée. Le problème se complique considérablement si la bactériocine doit être produite in situ, au cours d'un processus fermentaire. Puisque le transfert des gènes entre bactéries n'appartenant pas à la même espèce n'est pas encore autorisé, le choix des souches productrices de bactériocines se restreint à celles qui se développent bien dans ces processus. Bien que le choix de bactériocines soit déjà grand, il n'est pas sûr qu'une seule bactériocine possède toutes les propriétés requises. II faut donc envisager l'uti- lisation combinée de plusieurs de ces substances, ce qui peut rendre le problème pratiquement insoluble si elles doivent être produites in situ.

Une autre considération à prendre en compte, dans le cas de l'utilisation de bactériocines en routine, est le risque de faire apparaître dans l'environnement, et en particulier dans l'usine, des souches de bactéries indésirables résistantes à une ou plusieurs bactériocines. Avant d'envisager une telle utilisation des bactériocines, il serait donc prudent d'évaluer ce risque, et de déterminer de quelle manière on peut le limiter. II faudrait en particulier établir par quel(s) mécanisme(s) les bactéries cibles sont devenues résistantes, et étudier leur comportement dans l'environnement (possibilité de colonisation, stabilité du caractère, possibilité de transfert, dans l'environnement, des gènes codant pour ce caractère à d'autres bactéries...). D'ores et déjà on peut prédire que ce risque sera d'autant plus réduit que le niveau de contamination des aliments est faible et que l'efficacité de la bactériocine est plus grande, grâce à une utilisation rationnelle. C'est ce à quoi nous nous efforçons dans l'Unité de Recherches Laitières du centre INRA de Jouy-en-Josas, en cherchant à identifier les facteurs affectant l'activité des bactériocines au cours de la fabrication et de l'affinage des fromages à pâte molle.

Par ailleurs, les recherches menées dans divers laboratoires concernant l'organisation des gènes codant pour la production de bactériocine et leur régulation, le mode d'action de ces substances, et en particulier la relation structure-fonction, et les progrès de l'ingéniérie des protéines permettent d'envisager à plus long terme, la production, par des bactéries appropriées, de bactériocines parfaitement adaptées à un usage particulier. Cela n'exclut pas la production de peptides ayant les mêmes propriétés antibactériennes à partir de protéines naturelles, comme c'est le cas avec la lactoferricine B, 
issue de l'hydrolyse pepsique de la lactoferrine bovine (Tomita et al, 1991).

\section{REMERCIEMENTS}

Je remercie N Deschamps, P Emin, JJ Gratadoux, S Maisnier-Patin, M Murray, $\mathrm{H}$ Siswanto et HJ Song, pour leur contribution aux travaux réalisés au sein de l'Unité de recherches laitières.

\section{RÉFÉRENCES}

Beckers HJ, Soentoro PSS, Delfgou-van Asch EHM (1987) The occurrence of Listeria monocytogenes in soft cheeses and raw milk and its resistance to heat. Int J Food Microbiol 4, 249-256

Benkerroum N, Sandine WE (1988) Inhibitory action of nisin against Listeria monocytogenes. J Dairy Sci $71,3237-3245$

Bhunia AK, Johnson MC, Ray B (1988) Purification, characterization and antimicrobial spectrum of a bacteriocin produced by Pediococcus acidilactici. J Appl Bacteriol 65, 261-268

Delves-Broughton J (1990) Nisin and its application as food preservative. J Soc Dairy Technol 43, 7376

Fenlon DR, Wilson J (1989) The incidence of Listeria monocytogenes in raw milk from farm bulk tanks in North-East Scotland. J Appl Bacteriol 66, 191 196

Héchard Y, Derijard B, Letellier F, Cenatiempo Y (1992) Characterization and purification of mesentericin $Y$ 105, an anti-Listeria bacteriocin from Leuconostoc mesenteroides. J Gen Microbiol 138, 2725-2731

Hugenholtz J, de Veer GJCM (1991) Application of nisin $A$ and nisin $Z$ in dairy technology. In: Nisin and Novel Lantibiotics (Jung G, Sahl HG, eds) ESCOM, Leiden, Pays Bas

Jack RW, Tagg JR, Ray B (1995) Bacteriocin of Grampositive bacteria. Microbiol Rev 59, 171-200
Lovett J, Francis DW, Hunt JM (1987) Listeria monocytogenes in raw milk: Detection, incidence and pathogenicity. J Food Prot 50, 188-192

Maisnier-Patin S (1994) Potentiel inhibiteur de la nisine et de deux autres substances antibactériennes visà-vis de Listeria monocytogenes et problèmes soulevés par leur utilisation en technologie fromagère. Thèse de l'Université Claude-Bernard - Lyon I

Maisnier-Patin S, Deschamps N, Tatini SR, Richard J (1992) Inhibition of Listeria monocytogenes in Camembert cheese made with a nisin-producing starter. Lait 72, 249-263

Maisnier-Patin S, Tatini SR, Richard J (1995) Combined effect of nisin and moderate heat on destruction of Listeria monocytogenes in milk. Lait 75, 81 91

Peeler JT, Bunning VK (1994) Hazard assessment of Listeria monocytogenes in the processing of bovine milk. J Food Prot 57, 689-697

Pucci MJ, Vedamuthu ER, Kunka BS, Vanderbergh PA (1988) Inhibition of Listeria monocytogenes by using bacteriocin PA-1 produced by Pediococcus acidilactici PAC1.0. Appl Environ Microbiol 54, 2349-2353

Rohrbach BW, Draughon FA, Davidson PM, Oliver SP (1992) Prevalence of Listeria monocytogenes, Campylobacter jejuni, Yersinia enterolitica, and Salmonella in bulk tank milk: risk factors and risk of human exposure. J Food Prot 55, 93-97

Ryser ET, Marth EH (1987) Fate of Listeria monocytogenes during the manufacture and ripening of Camembert cheese. J Food Prot 50, 372-378

Schlyter JH, Glass KA, Loeffelholz J, Degnan AJ, Luchansky JB (1993) The effect of diacetate with nitrite, lactate, or pediocin on the viability of Listeria monocytogenes in turkey slurries. Int J Food Microbiol 19, 271-281

Siswanto HP (1993) Inhibition des listeria dans le lait de fabrication et à la surface des fromages à pâte molle et croûte lavée. Thèse de l'Université de Caen

Siswanto HP, Richard J (1992) Vitesse de croissance dans le lait de Listeria monocytogenes et autres souches du même genre à des températures suboptimales. Lait 72, 265-275

Tomita M, Bellamy W, Takase M, Yamauchi K, Wakashi H, Kawase K (1991) Potent antibacterial peptide generated by pepsin digestion of bovine lactoferrin. J Dairy Sci 74, 4137-4142 